Herz 2012 · 37:38-43

DOI 10.1007/s00059-011-3571-z

Online publiziert: 19. Januar 2012

๑) Urban \& Vogel 2012

\section{Einleitung}

Die kardiovaskulären Erkrankungen sind auch in der Schweiz die führende Ursache von Todesfällen und Hospitalisationen; die Bedeutung der direkten und indirekten sozioökonomischen Konsequenzen ist somit groß. Dies trotz Fortschritten bei den Erkenntnissen bezüglich pathophysiologischer Zusammenhänge, der Anwendung immer besserer diagnostischer Methoden und der ständigen Verbesserung der therapeutischen Möglichkeiten. Aufgrund dieser Tatsachen haben sich die Strukturen der kardiovaskulären Prävention und Rehabilitation auch in der Schweiz in den letzten Jahren rasch entwickelt. Die Anliegen der kardialen Rehabilitation werden in der Schweiz durch die Schweizerische Arbeitsgruppe für Kardiale Rehabilitation (SAKR) vertreten. Ziel dieser Arbeit ist es, die Entwicklung der strukturierten kardialen Rehabilitation in der Schweiz aufzuzeigen.

\section{Entwicklung der kardialen Rehabilitation in der Schweiz}

Die zunehmende Verbreitung der aktiven Rehabilitation von Herzpatienten geht auch in der Schweiz auf die späten 60er-Jahre zurück, als sich die Erkenntnis durchzusetzen begann, dass ein aufbauendes körperliches Training für Patienten nach Myokardinfarkt in verschiedener Hinsicht vorteilhaft ist [1]. Die strukturierte kardiale Rehabilitation wurde in der Schweiz anfänglich ausschließlich in Rehabilitationskliniken durchgeführt.

\title{
H. Saner
}

Klinik und Poliklinik für Kardiologie, Kardiovaskuläre Prävention und Rehabilitation, Inselspital Bern, Bern

\section{Der Schweizer Weg der kardiologischen Rehabilitation}

1972 wurden die ersten ambulanten Rehabilitationsprogramme für Herzpatienten in Bern und Zürich angeboten. Sie bestanden neben der hausärztlichen Betreuung/Begleitung mit Anpassung der medikamentösen Therapie, ergometrischen Kontrollen und Gesprächen über berufliche sowie soziale Probleme in 3 Gymnastikstunden pro Woche, die durch Physiotherapeuten unter Oberaufsicht eines Kardiologen geleitet wurden. In den folgenden Jahren entwickelten sich, bedingt durch die zunehmende Zahl von Herzpatienten, neben der steigenden Zahl von stationären Rehabilitationszentren immer mehr ambulante Rehabilitationsprogramme [2, 3].

Im Jahre 1985 wurde die SAKR als offizielle Arbeitsgruppe der Schweizerischen Gesellschaft für Kardiologie (SGK) gegründet. Eine ihrer ersten Aufgaben bestand in einer Erhebung der aktuellen Situation der kardialen Rehabilitation in der Schweiz mit dem Ziel, die daraus gewonnenen Kenntnisse für die Formulierung eines Anforderungsprofils für offiziell ankerkannte Institutionen der kardialen Rehabilitation zu erarbeiten und anhand dieser Kriterien später ein offizielles Verzeichnis dieser Institutionen zu erstellen. Im Jahre 1989 konnten bei einer Gesamtbevölkerung von rund 6,5 Mio. 39 Rehabilitationszentren für Herzpatienten mit total 42 Rehabilitationsprogrammen erfasst werden. Davon wurden 21 Programme stationär und 21 ambulant durchgeführt [4].

Im Jahre 1992 formulierte die SAKR erstmals Qualitätskriterien für ambulante und stationäre Rehabilitationszen- tren, die von den Kostenträgern als Voraussetzung für eine Kostenerstattung für das Rehabilitationsprogramm akzeptiert und in die entsprechende Leistungsverordnung des Bundes aufgenommen wurden. Die Qualitätskriterien basieren auf den Richtlinien und Empfehlungen der europäischen und amerikanischen Fachgesellschaften $[5,6,7,8,9]$. Das Anforderungsprofil definiert die verantwortliche ärztliche Leitung durch einen Kardiologen FMH, die Einrichtung inkl. Alarmkonzept, Häufigkeit und Inhalte von $\mathrm{Pa}$ tientenuntersuchungen, die Definition eines speziellen Programms für Herzpatienten inkl. strukturierter Bewegungstherapie, Entspannung und Sekundärprophylaxe mit Lebensstilinterventionen, obligatorische Inhalte des Abschlussberichts sowie eine permanente Qualitätskontrolle neben einer Mindestzahl von Herzpatienten pro Jahr insgesamt resp. nach akutem Krankheitsereignis. Diese Qualitätskriterien werden laufend überprüft und wurden in den letzten Jahren etwa alle 2 Jahre verfeinert. Aufgrund dieser Qualitätskriterien und der Schließung verschiedener Höhenkliniken wegen mangelnden Bedarfs bzw. ungenügender Rendite ist die Zahl der stationären kardialen Rehabilitationszentren von 25 im Jahr 1989 auf 11 im Jahr 2011 zurückgegangen, während die Zahl der ambulanten Programme von 21 auf über 50 zugenommen hat.

Die kardiovaskuläre Rehabilitation und Sekundärprävention spielt sich in auch in der Schweiz in 3 Phasen ab:

- Die erste Phase beginnt im Spital nach dem Akutereignis (Phase I); 


\begin{tabular}{l}
$\begin{array}{l}\text { Tab. } 1 \text { Obligatorische Inhalte eines kar- } \\
\text { dialen Rehabilitationsprogramms in der } \\
\text { Schweiz }\end{array}$ \\
\hline Medizinische Evaluation \\
\hline Kontrollierte körperliche Aktivität \\
\hline $\begin{array}{l}\text { Bekämpfung der kardiovaskulären Risiko- } \\
\text { faktoren }\end{array}$ \\
\hline Anleitung zu einem herzgesunden Lebensstil \\
\hline Stressmanagement \\
\hline $\begin{array}{l}\text { Psychosoziale Betreuung und praktische Rat- } \\
\text { schläge für die soziale und berufliche Wieder- } \\
\text { eingliederung }\end{array}$ \\
\hline
\end{tabular}

- nach dem Spitalaustritt erfolgt der Übertritt in eine spezialisierte Institution mit einem strukturierten ambulanten oder stationären Intensivprogramm (Phase II);

- der Langzeiteffekt resp. die Nachhaltigkeit der in der Phase II erzielten Ergebnisse wird gesichert durch die Langzeitrehabilitation z. B. in Herzgruppen (Phase III).

In der Schweiz werden aber auch zunehmend ambulante Spezialprogramme für spezifische Patientengruppen angeboten. Dies aufgrund zunehmender Evidenz, dass insbesondere Patienten mit Herzinsuffizienz von einer spezialisierten kardialen Rehabilitation profitieren können $[10,11]$.

Die obligatorischen Inhalte eines kardialen Rehabilitationsprogramms sind in - Tab. 1 dargestellt.

\section{Indikationen und Kontraindikationen}

Die kardiovaskuläre Rehabilitation ist in der Schweiz wie in den umliegenden Ländern in der Regel in folgenden Situationen indiziert:

- Patienten mit koronarer Herzkrankheit nach Myokardinfarkt, nach koronarer Revaskularisationsoperation oder Koronardilatation, Patienten mit medikamentös stabilisierter Angina pectoris;

- Patienten mit St. n. operativen Interventionen am Herzen oder den großen Gefäßen (z. B. nach Herzklappenoperationen und Aortendissektion);
- Patienten mit Myokarderkrankungen (z. B. dilatative, ischämische oder hypertensive Herzkrankheit) mit oder ohne Herzinsuffizienz;

- Patienten mit peripher-arterieller Verschlusskrankheit Stadium nach Fontaine II a/b.

$\mathrm{Zu}$ den Kontraindikationen der kardiovaskulären Rehabilitation gehören:

- maligne, nichtkontrollierte ventrikuläre Rhythmusstörungen;

- manifeste, nichtkontrollierte Herzinsuffizienz;

- Lungenerkrankung mit ausgeprägter Hypoxie, die auch mit Sauerstoffgabe nicht ausreichend zu kompensieren ist;

- Zusatzerkrankungen, welche durch körperliche Aktivität verschlechtert werden oder eine solche verunmöglichen;

- schwerwiegende muskuloskelettale Probleme mit geringem Autonomie- und damit auch Wiedereingliederungspotenzial;

- schwere Psychopathie oder Suchtkrankheit mit manifester, nichtkontrollierter Abhängigkeit

\section{Verordnung der kardiovaskulären Rehabilitation}

Die Verordnung einer kardiovaskulären Rehabilitation gehört auch in der Schweiz in die Hand des behandelnden Arztes und muss die spezifischen Bedürfnisse des einzelnen Patienten berücksichtigen. Die wichtigsten Voraussetzungen dazu sind:

- Motivation des Patienten zur Teilnahme am Programm;

- Möglichkeiten des Patienten, die Informationsangebote zu verstehen;

- die Chance zur Teilnahme an einem angepassten körperlichen Aktivitätsprogramm resp. das Fehlen von Kontraindikationen dazu.

Die vorgängige Durchführung eines Belastungstests erlaubt einerseits das Erkennen von Risikopatienten und andererseits die Planung eines optimalen Trainings. Die Wahl des Rehabilitationsmodus muss in den Händen des behandelnden Arztes bleiben, welcher Vorteile und Nachteile der einzelnen Rehabili- tationsformen kennen sollte. Eine komplette Liste der kardiovaskulären Rehabilitationszentren in der Schweiz ist auch auf der Website der Schweizerischen Herzstiftung (http://www.swissheart.ch $\rightarrow$ Rehabilitation $\rightarrow$ Herzerkrankungen $\rightarrow$ ambulante resp. stationäre Rehabilitation) abrufbar.

\section{Stationäre Prävention versus ambulante Rehabilitation}

Im Prinzip gibt es 2 Möglichkeiten, die Phase II der kardiovaskulären Rehabilitation durchzuführen, und zwar entweder ambulant oder stationär. Unabhängig davon, ob das Programm ambulant oder stationär ist, sind die Rehabilitationsziele im Grundsatz die gleichen. Aufgrund ihrer speziellen Struktur ermöglichen die stationären Programme in der Regel eine intensivere und komplexere Rehabilitation. Falls keine medizinischen Gründe für die Verschreibung einer stationären Rehabilitation vorliegen, wird eine ambulante kardiovaskuläre Rehabilitation in einem anerkannten Zentrum empfohlen. In ausgewählten Fällen kann auch eine Kombination dieser beiden Rehabilitationsformen eine ideale Lösung darstellen (intensiver verkürzter stationärer Beginn, gefolgt von einer ambulanten Programmphase). In jedem Fall soll aber eine möglichst große Nachhaltigkeit der eingeleiteten sekundärpräventiven Maßnahmen angestrebt werden durch die Empfehlung zur Teilnahme in einer Herzgruppe. Nur eine längerfristig anhaltende Lebensstilmodifikation mit fortgesetzter regelmäßiger körperlicher Aktivität erlaubt es, die erzielten Verbesserungen auch in eine langfristige Verbesserung der Prognose umzusetzen $[12,13,14,15,16]$.

Die individuelle Indikationsstellung zu einer stationären oder ambulanten kardiovaskulären Rehabilitation ist abhängig von folgenden Faktoren:

- Mobilität des Patienten,

- Schweregrad der kardiovaskulären

Erkrankung und der damit verbundenen körperlichen Beeinträchtigung,

- subjektive Wahrnehmung des eigenen Gesundheitszustands,

- soziale und berufliche Situation, 
- Existenz oder Fehlen eines adäquaten ambulanten Rehabilitationsprogramms in der Nähe des Wohnorts.

Die möglichen Vorteile einer stationären Rehabilitation sind in $\bullet$ Tab. 2 und die Vorteile einer ambulanten Rehabilitation in - Tab. 3 dargestellt.

Eine stationäre kardiovaskuläre Rehabilitation soll im Allgemeinen in folgenden Fällen in Betracht gezogen werden:

- komplexe medizinische, psychische, soziale, berufliche oder präventive Rehabilitation;

- Patienten mit sehr geringer körperlicher Leistungsfähigkeit;

- kein ambulantes, den Bedürfnissen des Patienten angepasstes Rehabilitationsprogramm in der Nähe des Wohnorts.

Eine ambulante Rehabilitation ist im Prinzip angezeigt, wenn keine spezielle Indikation für eine stationäre Rehabilitation vorliegt.

\section{Dauer der kardiovaskulären Rehabilitation}

Die ambulante Rehabilitation dauert in der Regel 12 Wochen [17]. Es existieren auch längere Programme mit progressivem Übergang in eine Herzgruppe. Die stationäre Rehabilitation dauert in der Regel 4 Wochen. In einzelnen Fällen kann sie auf 3 Wochen verkürzt werden; gelegentlich muss sie aber auch um eine oder mehrere Wochen verlängert werden.

\section{Herzgruppen (Phase III)}

Der Eintritt in eine Herzgruppe erlaubt im Allgemeinen eine bessere Nachhaltigkeit der in der Phase II erreichten Lebensstilveränderungen. Sie erlaubt, die Langzeitprognose zu verbessern, und sollte deshalb empfohlen werden. Die Herzgruppen sind in der Schweiz in Form von selbstverwalteten Gruppen unter der Schirmherrschaft und mit Unterstützung der Schweizerischen Herzstiftung organisiert. Die Mitglieder bezahlen die Teilnahme am wöchentlichen Training selbst. Das Training wird von einem speziell ausgebil-

Herz 2012 · 37:38-43 DOI 10.1007/s00059-011-3571-z

(c) Urban \& Vogel 2012

H. Saner

\section{Der Schweizer Weg der kardiologischen Rehabilitation}

\section{Zusammenfassung}

Seit den späten 60er-Jahren wird auch in der Schweiz die strukturierte kardiale Rehabilitation angeboten. Diese erfolgte anfänglich ausschließlich in Rehabilitationskliniken. 1972 wurden die ersten ambulanten Rehabilitationsprogramme für Herzpatienten in Zürich und Bern angeboten. In den folgenden Jahren entwickelten sich neben der steigenden Zahl von stationären Rehabilitationszentren immer mehr ambulante Rehabilitationsprogramme, vor allem in den größeren Bevölkerungsgebieten des deutschsprachigen Mittellandes und in der französischsprachigen Schweiz. Im Jahr 1985 hat die Schweizerische Arbeitsgruppe für Kardiale Rehabilitation (SAKR) als offizielle Arbeitsgruppe der Schweizerischen Gesellschaft für Kardiologie ihre Tätigkeit aufgenommen und als eine ihrer ersten Aufgaben eine Bestandsaufnahme der Institutionen der kardialen Rehabilitation in der Schweiz gemacht. Im Jahr 1989 gab es bei einer Gesamtbevölkerung von rund 6,5 Mio. 42 Rehabilitationsprogramme, wovon 21 Programme stationär und 21 ambulant durchgeführt wurden, allerdings mit rund $90 \%$ der Patienten in stationären Rehabilitationsprogrammen. 1992 hat die SAKR erstmals Qualitätskriterien im Sinne eines Anforderungsprofils für Institutionen der kardialen Rehabilitation in der Schweiz aufgestellt. Die Zahl der stationären Rehabilitationszentren ist zwischen 1989 und 2011 von 21 auf 11 zurückgegangen, während die Zahl der ambulanten Programme von 21 auf 51 zugenommen hat. Die ambulanten Rehabilitationszentren werden teilweise durch lokale Ärztegruppen organisiert, mehrheitlich sind sie aber an Spitäler angegliedert. Für die Zukunft ist ein anhaltender Trend zur Verschiebung von der stationären zur ambulanten Rehabilitation zu erwarten. Dafür werden immer mehr ältere polymorbide Herzpatienten stationäre Rehabilitationsprogramme beanspruchen.

\section{Schlüsselwörter}

Kardiale Rehabilitation - Sekundärprävention . Qualitätskriterien · Audit

\section{The Swiss approach for cardiac rehabilitation}

\section{Abstract}

Structured cardiac rehabilitation goes back to the late 1960s also in Switzerland and in the beginning was only available in rehabilitation clinics. In 1972 the first ambulatory rehabilitation programs became available to patients in Zurich and Bern. In the following years, in addition to the increasing number of rehabilitation centers for inpatients, more and more ambulatory rehabilitation programs were developed, especially in the larger Midlands population area in German and French-speaking Switzerland. In 1985 the Swiss Working Group of Cardiac Rehabilitation (SAKR) was initiated as an official working group of the Swiss Society of Cardiology and one of its first tasks was to establish a list of the institutions for cardiac rehabilitation in Switzerland. At that time there were 42 rehabilitation programs for a population of approx. 6.5 million, 21 for inpatients and 21 ambulatory; however, $90 \%$ of the patients were in inpatient programs. In 1992 the SAKR group defined the quality criteria which were to be applied for official recognition of institutions for cardiac rehabilitation in Switzerland. Due to these criteria, plus the fact that an increasing number of rehabilitation clinics in the mountains had been closed down, the number of inpatient rehabilitation centers decreased from 21 to 11 between 1989 and 2011, whereas the number of ambulatory programs increased from 21 to 51 . The ambulatory rehabilitation centers are partially organized by local medical groups; however, most have integrated their activities into the local hospitals. The trend shows a developing preference for ambulatory rehabilitation. More and more elderly, polymorbid patients, however, will still need care in inpatient programs.

Keywords

Cardiac rehabilitation - Secondary prevention · Quality criteria · Audit deten Sportlehrer oder Physiotherapeuten geleitet. Der freiwillige ärztliche Herzgruppenleiter steht beratend zur Seite, ist aber beim Training nicht anwesend. Eine Liste der Herzgruppen in der Schweiz ist einsehbar auf der Website der Schweizerischen Herzstiftung (http://www.swissheart.ch $\rightarrow$ Herzerkrankungen $\rightarrow$ Herzgruppen). 


\section{Tab. 2 Mögliche Vorteile einer stationären Rehabilitation}

Möglichkeit eines früheren Programmbeginns nach dem Akutereignis

Betreuung von komplexen Krankheitsbildern mit erhöhtem Komplikationsrisiko und noch fehlender klinischer Stabilität

Aufnahme von älteren und behinderten Patienten, v. a. auch mit schwerwiegenderen Komorbiditäten

Intensivierte Maßnahmen zu einem möglichst reibungslosen Wiedereintritt in das tägliche Leben zur Vermeidung von Hilfsbedürftigkeiten und Abhängigkeiten bei der Alltagsbewältigung zu Hause

Rasche Wiederaufnahme der vollen Erwerbstätigkeit

Tab. 3 Mögliche Vorteile einer ambulanten Rehabilitation

Erholung in der gewohnten Umgebung

Einbezug der Familie/des Partners in die Modifikation des Lebensstils

Aufzeigen von Aktivitätsmöglichkeiten in der Umgebung des Wohnorts

Möglichkeit einer unmittelbaren Wiederaufnahme einer teilzeitigen Arbeitstätigkeit

Erleichterung einer Überführung in eine lokale Herzgruppe

\section{Anforderungsprofil für von der SAKR offiziell anerkannte Rehabilitationskliniken/- institutionen (Februar 2011)}

Damit die Ziele der kardiovaskulären Rehabilitation erreicht und eine fachlich optimale Betreuung der Patienten sichergestellt werden können, muss ein solches Programm gewisse Qualitätskriterien erfüllen. Diese sind für die Schweiz von der SAKR auf der Basis internationaler Empfehlungen und Richtlinien und dabei insbesondere der Positionspapiere der Sektion kardiale Rehabilitation der European Association for Cardiovascular Prevention and Rehabilitation ausgearbeitet und falls nötig an spezielle Gegebenheiten in unserem Land adaptiert worden; die Anerkennung einer kardiovaskulären Rehabilitationsinstitution als Leistungserbringer zu Lasten der obligatorischen Krankenversicherung bedingt die Erfüllung dieser Qualitätskriterien und die daraus resultierende offizielle Anerkennung [gemäß KLV (832.112.31), Anhang 1, Kapitel 11].

\section{1. Ärztliche Leitung des \\ Rehabilitationsprogramms}

Die verantwortliche ärztliche Leitung erfolgt durch einen in Rehabilitation erfahrenen Kardiologen (geregelte Vertretung, regelmäßige Zeit verfügbar), für stationäre Programme durch einen festangestellten (zu mind. 2 Drittel) Facharzt für Kar- diologie mit adäquater Ausbildung in kardiovaskulärer Rehabilitation und zusätzlicher internistischer Ausbildung, welche beide jeweils sowohl für das Rehabilitationsprogramm als auch für das Rehabilitationsteam (Führung und Ausbildung) verantwortlich sind. In Anbetracht der häufig multimorbiden Patienten mit ausgeprägtem Risikoprofil muss der ein stationäres Rehabilitationsprogramm leitende Kardiologe auch eine internistische Ausbildung von mindestens 3 Jahren vorweisen können.

\section{Einrichtung der Rehabilitationsinstitutionen}

\subsection{Alarmkonzept für Notfälle} inkl. unmittelbarer Reanimationsbereitschaft obligatorisch

Bewegungstherapeuten, Ärzte, Pflegefachkräfte und anderes beteiligtes Fachpersonal müssen regelmäßig (4-mal jährlich) in Reanimationsmaßnahmen geschult werden. Es muss sichergestellt werden, dass die primäre Reanimation (CPR, „cardiopulmonary resuscitation") sofort und die erweiterte Reanimation (ACLS, "advanced cardiac life support") innerhalb einer Frist von 4 min begonnen werden kann. Bei Aktivitäten im Gelände gelten die gleichen Konditionen, wobei als ACLS-Maßnahme eine Defibrillation möglich sein muss. Dies bedingt, dass ein entsprechendes Gerät mitgeführt wird und dass 2 in CPR und Anwendung des Defibrillators geschulte Personen wäh- rend der Trainingsaktivitäten anwesend sind.

\subsection{Komplettes Reanimations- material inkl. Defibrillator, wenn möglich transkutaner Pacemaker}

\subsection{Mehrkanal-EKG}

\subsection{Ergometrieplatz mit Fahrradergometer oder Laufbandergometer}

\subsection{2-D-Doppler-Echokardiographie an der Institution verfügbar}

\subsection{Langzeit-EKG (Auswertung an der Institution selbst oder durch einen regelmäßig verfügbaren Partner) oder evtl. Telemetrie}

\section{Patientenuntersuchung}

\subsection{Eintrittsuntersuchung}

Die Eintrittsuntersuchung muss eine allgemeine internistische Untersuchung inklusive einen maximal symptomlimitierten Belastungstest am Fahrradergometer oder am Laufbandergometer enthalten, falls ein solcher nicht innerhalb der letzten 3 Wochen vor Beginn eines aktiven Bewegungsprogramms durchgeführt wurde. Zu Beginn der Rehabilitation muss ein Risikofaktorenprofil vorhanden sein. Ist im stationären Bereich der Patient nicht in der Lage, eine Ergometrie zu absolvieren, kann diese durch einen 6-Minuten-Gehtest oder einen anderen geeigneten Funktionstest ersetzt werden.

\subsection{Austrittsuntersuchung}

Es wird eine erneute klinische kardiopulmonale Untersuchung inkl. Ergometrie gefordert. Im Weiteren sollte das $\mathrm{Ri}$ sikofaktorenprofil erneut überprüft werden. Ist im stationären Bereich der Patient nicht in der Lage, eine Ergometrie zu absolvieren, kann diese durch einen 6-Minuten-Gehtest oder einen anderen geeigneten Funktionstest ersetzt werden.

\section{Spezifisches Programm für Herzpatienten}

Die Rehabilitationsinstitution muss ein spezielles Programm für die Rehabilita- 
tion von Herzpatienten haben. Der Inhalt dieses Programms richtet sich nach den jeweils gültigen europäischen Richtlinien (aktuell [9]). Zusätzlich gelten folgende Punkte:

- Leitung der Bewegungstherapie durch speziell ausgebildete Herztherapeuten/innen (SAKR oder eine äquivalente, von der SAKR anerkannte Ausbildung), die während der Aktivitäten in der Regel präsent sein müssen. Die Mitglieder des Behandlungsteams müssen regelmäßig an den Weiterbildungen der SAKR oder an gleichwertigen Ausbildungsgängen teilnehmen.

- Um dem unterschiedlichen Leistungsvermögen der Teilnehmer gerecht zu werden, sollten die Programmteile, die eine einheitliche Leistung erfordern (z. B. Gymnastik oder Wanderungen) in stationären Programmen in mind. 3 Leistungsstärken angeboten werden. In ambulanten Programmen sind mehrere Leistungsgruppen nicht erforderlich.

\section{Erstellen eines Abschlussberichts}

Der Abschlussbericht muss mindestens die folgenden Angaben enthalten:

- Bericht über den Rehabilitationsverlauf, Resultate der Eintritts- und der Abschlussuntersuchung inkl. Resultate des Belastungstests;

- spezielle physische und psychische Aspekte des Patienten;

- Risikofaktoren;

- aktuelle medikamentöse Therapie;

- Vorschläge für weitere diagnostische und therapeutische Maßnahmen

- Arbeitsfähigkeit.

Der Abschlussbericht muss durch einen Arzt verfasst sein.

\section{Permanente Qualitätskontrollen}

\subsection{Qualitätskriterien}

\section{(Anforderungsprofil)}

Das Angebot der Programme wird durch einen jährlich auszufüllenden Fragebogen und bei Aufnahme durch ein Audit überprüft. Institutionen, die die geforderten Qualitätskriterien nicht erfüllen, können nicht als von der SAKR/SGK anerkannte
Institution aufgenommen werden. Bei bereits akkreditierten Programmen besteht bei Nichterfüllen der Bedingungen eine Frist von 1 Jahr bis zur Streichung.

\subsection{SAKR-Statistik}

Einmal pro Jahr wird obligatorisch eine Patientenstatistik ausgefüllt, die über die Zahl der Patienten und der Hauptdiagnosen Auskunft gibt und insbesondere die Frage der während der Rehabilitation aufgetretenen Komplikationen beantwortet.

\subsection{Regelmäßige Erhebung der Patientenzufriedenheit nach anerkannten Methoden}

\subsection{Patientenzahlen}

6.4.1 Stationäre Rehabilitationszentren. Anforderungen: mindestens 200 Herzpatienten pro Jahr, davon mindestens 2 Drittel nach akutem Krankheitsereignis

6.4.2 Ambulante Rehabilitationsprogramme. Anforderungen: mindestens 50 Herzpatienten pro Jahr nach akutem Krankheitsereignis. Da neu angebotene Rehabilitationsprogramme in der Regel in der ersten Phase die geforderten Patientenzahlen nicht erreichen, ist eine provisorische Aufnahme möglich. Entsprechende Programme werden im Verzeichnis als „Programm im Aufbau“ gekennzeichnet. Die geforderten Patientenzahlen sollten innerhalb von $1 \mathrm{Jahr}$ erreicht werden.

\section{Von der Rehabilitation zur Prävention}

Heute gilt es als allgemein anerkannt, dass es sich bei der Arteriosklerose um einen lebenslangen Prozess bei Patienten handelt, die kardiovaskuläre Risikofaktoren aufweisen und genetisch nicht dagegen geschützt sind. Auf der einen Seite muss deshalb der Schritt von der Sekundär- zur Primärprävention deutlich früher im Leben erfolgen (was zukünftig durch verschiedene Techniken der Früherkennung der Arteriosklerose inklusive bildgebender Verfahren und Serummarker möglich sein wird). Auf der anderen Seite hat sich die kardiale Rehabilitation der neu- en Herausforderung zu stellen und muss ihre Hauptaktivitäten weg von den früher dominierenden eigentlichen Rehabilitationsaspekten auf die neuen Bedürfnisse nach Verbesserung der (Langzeit-) Prävention ausrichten $[18,19]$. Dies umso mehr, als heute bei rund 85\% der Patienten die kardiale Rehabilitation nach einer Abklärung und/oder Intervention wegen koronarer Herzkrankheit erfolgt. Zudem ist die Mehrheit der Patienten nicht mehr wie früher durch eine längere Bettlägerigkeit dekonditioniert und für Komplikationen der Immobilität gefährdet, sondern verlässt das Spital wenige Tage nach der Intervention in gutem Allgemeinzustand.

Zentrale Ziele der Sekundärprävention im Rahmen eines kardiologischen Rehabilitationsprogramms sind neben der guten körperlichen Leistungsfähigkeit, dem psychischen Wohlbefinden und der sozialen Integration neu ein Stopp der Progression der Grundkrankheit und damit eine Reduktion der Rückfallrate resp. der Rehospitalisationen. Damit soll sowohl eine Verbesserung der Lebensqualität als auch eine Reduktion der Folgekosten erreicht werden. Aus diesem Grund sind in der Schweiz seit Kurzem auch ambulante Rehabilitationsprogramme für Patienten mit Diabetes Typ 2 und für Patienten mit peripher-arterieller Verschlusskrankheit Stadium nach Fontaine IIa/b bei Erfüllen der Qualitätskriterien für die Krankenkassen leistungspflichtig.

\section{Schlussfolgerungen}

Zusammenfassend können wir festhalten, dass sich die kardiale Rehabilitation in den letzten 50 Jahren in der rehabilitativen Betreuung von Herzpatienten in der Schweiz gut etabliert hat. Stationäre und ambulante Rehabilitationsformen sind bei korrekter Indikationsstellung komplementär. Insgesamt dürfen wir aber doch - nicht zuletzt auch aus ökonomischen Überlegungen heraus in den kommenden Jahren mit einem Trend zur Verschiebung von stationären zu ambulanten Rehabilitationszentren rechnen. Die ambulante Rehabilitation soll in der Regel 12 Wochen dauern, um einen Langzeiteffekt auf das Fortschreiten der Arteriosklerose erreichen zu kön- 
nen. Dafür werden die stationären Rehabilitationszentren zunehmend ältere polymorbide und damit intensiver pflege- und betreuungsbedürftige Patienten erhalten. Solche Überlegungen werden in der Diskussion mit Politik und Kostenträgern über die Zukunft der kardialen Rehabilitation in der Schweiz eine zentrale Rolle spielen.

\section{Korrespondenzadresse}

\section{Prof. Dr. H. Saner}

Klinik und Poliklinik für Kardiologie, Kardiovaskuläre Prävention und Rehabilitation, Inselspital Bern

CH-3010 Bern

Schweiz

hugo.saner@insel.ch

Interessenkonflikt. Der korrespondierende Autor weist auf folgende Beziehungen hin: Leiter eines ambulanten kardialen Rehabilitationsprogramms am Inselspital Bern.

\section{Literatur}

1. Saner H (Hrsg) (1993) Kardiale Rehabilitation. Thieme, Stuttgart

2. Saner H, Pfiffner D (1995) Ambulante Rehabilitation von Herzpatienten in der Schweiz. Wien Klin Wochenschr 107(24):771-773

3. Saner H (2002) Ambulante kardiale Rehabilitation in der Schweiz. Dtsch Z Sportmed 53:130-134

4. Pfiffner D, Saner H (1990) Aktuelle Situation der kardialen Rehabilitation in der Schweiz. Schweiz Med Wochenschr 120:1565-1568

5. Giannuzzi P, Saner H, Björnstad H et al (2003) Secondary prevention through cardiac rehabilitation: position paper of the working group on cardiac rehabilitation and exercise physiology of the European Society of Cardiology. Eur Heart J 24:12731278

6. Giannuzzi P, Mezzani A, Saner H et al (2003) Physical activity for primary and secondary prevention. Position paper of the working group on cardiac rehabilitation and exercise physiology of the European Society of Cardiology. Eur J Cardiovasc Prev Rehabil 10:319-327

7. Braunwald E, Antman EM, Beasley JW et al (2002) ACC/AHA 2002 guideline update for the management of patients with unstable angina and nonST-segment elevation myocardial infarction: summary article: a report of the American College of Cardiology/American Heart Association Task Force on Practice Guidelines. J Am Coll Cardiol 40:13661374
8. Balady GJ, Williams M, Ades PA et al (2007) Core components of cardiac rehabilitation/secondary prevention programs: 2007 update: a scientific statement from the American Heart Association Exercise, Cardiac Rehabilitation, and Prevention Committee, the Council on Clinical Cardiology; the Councils on Cardiovascular Nursing, Epidemiology and Prevention, and Nutrition, Physical Activity, and Metabolism; and the American Association of Cardiovascular and Pulmonary Rehabilitation. Circulation 115:2675-2682

9. Piepoli MF, Corrà U, Benzer W et al (2010) Secondary prevention through cardiac rehabilitation: from knowledge to implementation. A position paper from the Cardiac Rehabilitation Section of the European Association of Cardiovascular Prevention and Rehabilitation. Eur J Cardiovasc Prev Rehabil 17:1-17

10. Piepoli MF, Davos C, Francis DP, Coats AJ (2004) ExTraMATCH Collaborative. Exercise training metaanalysis of trials in patients with chronic heart failure (ExTraMATCH). BMJ 328:189-193

11. Schmid JP, Blatter-Bühler $P$, Gaillet R et al (2010) Impact of a cardiac rehabilitation programme on exercise capacity, parameters of left ventricular function and health-related quality of life in chronic heart failure patients. Results from a prospective single centre cohort. Cardiovascular Medicine 13:86-91

12. Saner H, Saner B, Stäubli R (1994) Erste Erfahrungen mit einem komprehensiven ambulanten Rehabilitationsprogramm für Herzpatienten. Schweiz Med Wochenschr 124:2075-2082

13. Saner H (1995) Kardiale Rehabilitation - wie und wo? Schweiz Med Wochenschr 125:1763-1765

14. Müller K, Saner H (2002) Wirksame und wirtschaftliche kardiale Rehabilitation: wissenschaftliche und konzeptionelle Grundlagen. Cardiovascular Medicine 5:222-234

15. Bjarnason-Wehrens B, McGee H, Zwisler AD et al (2010) Cardiac rehabilitation in Europe: results from the European Cardiac Rehabilitation Inventory Study. Eur J Cardiovasc Prev Rehabil 17:410-418

16. Mittag O, Schramm S, Böhmen S et al (2011) Medium-term effects of cardiac rehabilitation in Germany: systematic review and meta-analysis of results from national and international trials. Eur J Cardiovasc Prev Rehabil 18(4):587-593

17. Hammill BG, Curtis LH, Schulman KA, Whellan DJ (2010) Relationship between cardiac rehabilitation and long-term risks of death and myocardial infarction among elderly Medicare beneficiaries. Circulation 121:63-70

18. Saner H (2003) Von der kardialen Rehabilitation zur Prävention. Wien Klin Wochenschr 115/2122:743-744

19. http://www.heartcharter.eu/ 